\title{
A Novel Mutation of SLC19A2 in a Chinese Zhuang Ethnic Family with Thiamine- Responsive Megaloblastic Anemia
}

\author{
Xiaoying Xian ${ }^{\mathrm{a}}$ Lin Liao ${ }^{\mathrm{a}}$ Wei Shu ${ }^{\mathrm{b}}$ Hongtao Lic Yuanyuan Qin ${ }^{\mathrm{a}} \quad$ Jie Yan ${ }^{\mathrm{a}}$ \\ Jianming Luo ${ }^{d}$ Faquan Lin ${ }^{a}$ \\ aDepartment of Clinical Laboratory, The First Affiliated Hospital of Guangxi Medical University, Nanning, \\ bDepartment of Cell Biology and Genetics, Guangxi Medicinal University, Nanning, 'Scientific Research \\ Center of Guangxi Medical University, Nanning, dDepartment of Pediatrics, The First Affiliated Hospital \\ of Guangxi Medical University, Nanning, China
}

\section{Key Words}

Trma • Megaloblastic anemia • Diabetes mellitus • Visual impairment • SLC19A2 mutation

\begin{abstract}
Background/Aims: Thiamine-responsive megaloblastic anemia syndrome is a rare autosomal recessive disorder resulting from mutations in SLC19A2, and is mainly characterized by megaloblastic anemia, diabetes, and progressive sensorineural hearing loss. Methods: We study a Chinese Zhuang ethnicity family with thiamine-responsive megaloblastic anemia. The proband of the study presented with anemia and diabetes, similar to his late brother, as well as visual impairment. All clinical manifestations were corrected with thiamine $(30 \mathrm{mg} / \mathrm{d})$ supplementation for 1-3 months, except for visual impairment, which was irreversible. The presence of mutations in all exons and the flanking sequences of the SLC19A2 gene were analyzed in this family based on the proband's and his brother's clinical data. Computer analysis and prediction of the protein conformation of mutant THTR-1. The relative concentration of thiamine pyrophosphate in the proband's whole blood before and after initiation of thiamine supplement was measured by high performance liquid chromatography (HPLC). Results: Gene sequencing showed a homozygous mutation in exon 6 of the SLC19A2 gene (c.1409insT) in the proband. His parents and sister were diagnosed as heterozygous carriers of the c.1409insT mutation. Computer simulation showed that the mutations caused a change in protein conformation. HPLC results suggested that the relative concentration of thiamine pyrophosphate in the proband's whole blood after thiamine supplement was significantly different $(P=0.016)$ from that at baseline. Conclusions: This novel homozygous mutation (c.1409insT) caused the onset of thiamine-responsive megaloblastic anemia in the proband.
\end{abstract}

X. Xian and L. Liao contributed equally to this work.

\begin{tabular}{ll}
\hline Faquan Lin & Department of Clinical Laboratory, The First Affiliated Hospital of Guangxi Medical \\
& University Nanning, Guangxi (China) \\
& E-Mail fqlin1998@163.com
\end{tabular}

\section{KARGER}




\section{Introduction}

Thiamine-responsive megaloblastic anemia (TRMA) syndrome is a rare autosomal recessive disorder characterized by megaloblastic anemia, diabetes mellitus, and progressive sensorineural hearing loss [1]. In addition, there are some unusual manifestations, including stroke-like onset, thrombocytopenia, ataxia, liver enlargement, arrhythmia, retinal degeneration, and short stature [2,3]. These clinical manifestations, however, are unspecific, and genetic testing is believed to be a TRMA-specific diagnostic method. Mutations in SLC19A2 on chromosome 1q23.3, encoding thiamine transporter-1 comprising 497 amino acids, have been confirmed to cause TRMA syndrome [4]. To date, at least 43 mutations in the SLC19A2 gene have been reported to cause TRMA, most of which are missense and nonsense mutations followed by insertion and deletion mutations. In this study, we analyzed a Chinese Zhuang ethnic family with TRMA syndrome by PCR sequencing, and revealed the genetic pathogenesis in the onset of TRMA in the family, providing evidence for the diagnosis and treatment of this disease.

\section{Materials and Methods}

\section{Patients}

We studied a Chinese Zhuang ethnic family with TRMA syndrome. The proband's parents of Zhuang ethnicity from Nanning, Guangxi Zhuang Autonomous Region in China denied in-law marriage, a family history of disease and a history of intrauterine infection. The diagnosis was made based on clinical manifestations of megaloblastic anemia, diabetes, visual impairment, and genetic analysis.

The study procedure was approved by the Ethics Committee of the First Affiliated Hospital of Guangxi Medical University in China.

\section{Genotyping}

In accordance with the principle of informed consent, $2 \mathrm{~mL}$ of anticoagulant peripheral blood was extracted from each of the proband and his family members. A DNA extraction kit (TIANGEN, Beijing, China) was used to isolate genomic DNA from the peripheral blood samples. All SLC19A2 exons and their flanking intronic regions were amplified by a primer set designed based on the sequence of the SLC19A2 gene (NC_000001.11) in GenBank. The PCR reaction sample had a total volume of $30 \mu \mathrm{L}$ consisting of $10.6 \mu \mathrm{L}$ of double distilled water, $1.2 \mu \mathrm{L}$ of $10 \mathrm{nmol} / \mathrm{L}$ upstream and downstream primers, $15 \mu \mathrm{L}$ of $2 \times$ Taq PCR Master Mix (containing dye) (CWBIO, Beijing, China), and $2 \mu \mathrm{L}$ of DNA template. PCR conditions were as follows: initial denaturation at $94^{\circ} \mathrm{C}$ for 2 minutes, followed by 35 cycles of denaturation at $94^{\circ} \mathrm{C}$ for 30 seconds, annealing at $58^{\circ} \mathrm{C}$ for 30 seconds and elongation at $72^{\circ} \mathrm{C}$ for 1 minute, with a final elongation at $72^{\circ} \mathrm{C}$ for 2 minutes. Samples were stored at $4^{\circ} \mathrm{C}$ until use. PCR products were electrophoresed by $2 \%$ agarose gel electrophoresis for 30 minutes. The specificity of the amplified products was determined by gel imaging and then bi-directional sequencing was performed by BGI Co., Ltd., Shenzhen, China

\section{Bioinformatic analysis}

The THTR-1 transmembrane region was predicted using TMHMM Server v. 2.0 (http://www.cbs. dtu.dk/services/TMHMM/). The conservation of THTR-1 partial amino acid sequences in 10 species (human, pan troglodytes, nomascus leucogenys, macaca mulatta, bos taurus, sus scrofa, felis catus, canis lupus familiaris, mus musculus, rattus norvegicus) was analyzed by clustalx software. The THTR-1 threedimensional structure was predicted using the I-TASSER server (http://zhanglab.ccmb.med.umich.edu/ITASSER/] [5].

High performance liquid chromatography (HPLC) detection

HPLC was performed as previously described [6]. The main reagents included thiamine, thiamine monophosphate (TMP), thiamine pyrophosphate (TPP), potassium ferricyanide, disodium hydrogen phosphate, methyl tert-butyl ether, trichloroacetic acid (TCA) (Sigma, USA), and methanol (Merck, Germany), which were all chromatographically pure. Hydrochloric acid and sodium hydroxide were analytically pure. Ultra-pure water (Milli-Q Academic A10, Millipore, USA) was used in the experiment. 


\section{Cellular Physiology Cell Physiol Biochem 2018;47:1989-1997 \\ \begin{tabular}{l|l} 
and Biochemistry POI: 10.1159/000491467 & $\begin{array}{l}\text { (c) 2018 The Author(s). Published by S. Karger AG, Basel } \\
\text { www.karger.com/cpb }\end{array}$
\end{tabular}}

Xian et al.: Clinical Manifestations of a Novel Mutation of SLC19A2

The main instruments included a Waters2695 liquid chromatograph (Alliance System, Waters, MA, USA); an Agilent TC-C18 liquid chromatographic column $\left(4.6 \times 250 \mathrm{~mm}, 5 \mu \mathrm{m}, 25^{\circ} \mathrm{C}\right)$; methanol for mobile phase A, and $25 \mathrm{mmol} / \mathrm{L}$ disodium phosphate for mobile phase B; 10-25\%A (0-6 minutes), 25-45\%A (6-8 minutes), 45-10\%A (8-16minutes), 10\%A (16-20minutes) for gradient elution; flow rate: $1 \mathrm{~mL} / \mathrm{min}$; sample size: $10 \mu \mathrm{L}$; fluorescence excitation wavelength: $375 \mathrm{~nm}$, and emission wavelength: $435 \mathrm{~nm}$.

Preparation of standard samples: according to the molecular weight of thiamine, TMP and TPP, a $1 \mathrm{mmol} / \mathrm{L}$ stock solution $\left(0.1 \mathrm{~mol} / \mathrm{L}\right.$ hydrochloric acid as the solvent) was prepared and stored at $-80^{\circ} \mathrm{C}$. The stock solution was then diluted to a working solution with a concentration of $0,10,20,40,60$, or $80 \mathrm{nmol} / \mathrm{L}$ thiamine and TMP, as well as to a concentration of $0,100,200,400,600$, or $800 \mathrm{nmol} / \mathrm{L}$ TPP. The working solution was prepared immediately before use.

Sample treatment: EDTA anticoagulant samples were extracted from the proband (before supplementation with thiamine and after supplementation with thiamine for 3 months) and the healthy controls (six children with normal blood test results, aged 2-15 years, median age: 4.3 years), $500 \mu \mathrm{L}$ of which was taken and placed into a $1.5 \mathrm{~mL}$ EP tube and stored at $-80^{\circ} \mathrm{C}$. Blood samples were thawed at room temperature, and then the standard sample and water were added into $500 \mu \mathrm{L}$ of $10 \%$ TCA, shocked for 15 seconds, placed on ice for 30 minutes, and centrifuged at $4^{\circ} \mathrm{C}$ at $13,000 \times g$ for 6 minutes. Then the supernatant was filtered through a microporous filter of $0.22-\mu \mathrm{m}$ thickness, and mixed with $750 \mu \mathrm{L}$ methyl butyl ether to remove TCA after two rinses. Then, $80 \mu \mathrm{L}$ of the supernatant was mixed with $20 \mu \mathrm{L}$ of methanol and $50 \mu \mathrm{L}$ of freshly prepared $2 \times 10^{-4} \mathrm{~mol} / \mathrm{L}$ potassium ferricyanide ( $15 \%$ sodium hydroxide as solvent) and used after filtering through a $0.22-\mu \mathrm{m}$ microporous membrane.

\section{Statistical analysis}

The data were statistically analyzed using SPSS 22.0 (IBM Corp., Armonk, NY, USA), and measurement data were expressed as the mean \pm standard deviation and compared using the Student's $t$-test. $P<0.05$ was considered statistically significantly.

\section{Results}

\section{Clinical investigation}

The proband of the study was a 4-month-old boy of Chinese Zhuang ethnicity who was hospitalized for paroxysmal cough. At admission, he presented with skin and mucous pallor, but no abnormalities in the heart, chest, neck, or head. His laboratory tests were as follows. (1) Routine blood analysis: red blood cells (RBC): $2.47 \times 10^{12} / \mathrm{L}$, hemoglobin (Hb): $70.2 \mathrm{~g} / \mathrm{L}$, white blood cells (WBC): $7.82 \times 10^{9} / \mathrm{L}$, platelets: $199 \times 10^{9} / \mathrm{L}$, mean corpuscular volume (MCV): $88 \mathrm{fl}$, and hematocrit (HCT): 0.21. (2) Biochemical tests showed no functional abnormalities in the liver, kidney and electrolytes, the fasting blood glucose (FBG) level was $13.9 \mathrm{mmol} / \mathrm{L}$, and the lactate dehydrogenase (LD) level was 374U/L. (3) A peripheral blood smear test showed mature RBCs of different sizes that were large, oval or helmetshaped, partially presenting with an enlarged region indicated by weak staining in the center (Fig. 1A). (4) Active hyperplasia and developmental disorder, as well as occasional megaloblastic degeneration in the bone marrow were detected by bone marrow cytology (Fig. 1B). (5) Iron staining showed many extracellular iron particles and iron beads (Fig. 1C), 61\% intracellular iron, and occasional ring sideroblasts (Fig. 1D). (6) Color eye fundus images of the proband showed binocular macular defects (Fig. 2). (7) Results from optical coherence tomography (OCT) showed a thinned retina at the macular center (at recession), with the disappearance of the outer membrane (ELM) and inner segment/outer segment (IS/OS) layer (Fig. 2), indicating atrophy of the bilateral retina. (8) Auditory brainstem response (ABR) examination results showed the ABR threshold responding to the left and right air conduction was less than or equal to $30 \mathrm{dBnHL}$, indicating normal hearing function. The hearing test was conducted in a soundproof room, and both visual and hearing tests were performed when the proband was 20 months old. Considering these manifestations and laboratory test results, the proband was diagnosed with TRMA syndrome, and treated with thiamine (30 mg/d) for 1-3 months, which improved anemia and diabetes but visual impairment was irreversible. 


\section{Cellular Physiology Cell Physiol Biochem 2018;47:1989-1997 \begin{tabular}{c|c} 
DOI: 10.1159/000491467 & $\begin{array}{l}\text { O 2018 The Author(s). Published by S. Karger AG, Basel } \\
\text { www.karger.com/cpb }\end{array}$
\end{tabular} Xian et al.: Clinical Manifestations of a Novel Mutation of SLC19A2}

Fig. 1. Cell morphology examination of the proband (A-D) and his brother (E-F). A. A peripheral blood smear test showed mature red blood cells of different sizes, and macro erythrocytes were visible (Wright's staining, $\times 1000$ magnification). $\mathrm{B}$. Bone marrow cytology showed active hyperplasia and developmental disorder in the bone marrow, as well as occasional megaloblastic degeneration (Wright's staining, $\times 400$ magnification). C. Iron staining showed many extracellular iron particles and occasional iron beads $(\times 100$ magnification). D. Occasional ring sideroblasts were indicated by iron staining $(\times 1000$ magnification $)$. E. Bone marrow cytology showed active hyperplasia and a developmental disorder, megaloblastic degeneration in the bone marrow, and vacuoles in part of the erythroblastic cytoplasm (Wright's staining, $\times 1000$ magnification). F. Ring sideroblasts are indicated by iron staining $(\times 1000$ magnification).

The proband's late brother was also hospitalized for cough at 4 months of age. His parents confirmed that the brother presented with skin pallor and scattered petechiae. His examination results detected in another hospital were as follows: $\mathrm{Hb} 51 \mathrm{~g} / \mathrm{L}, \mathrm{FBG} 20.3$ $\mathrm{mmol} / \mathrm{L}$, megaloblastic degeneration with the presence of H-J corpuscles and ring sideroblasts in a bone marrow test. The proband's brother was diagnosed with sideroblastic anemia and neonatal diabetes mellitus at that time, and treated with blood transfusions and insulin treatment. In the following months, his blood glucose level showed an improvement (5.8 to $12 \mathrm{mmol} / \mathrm{L}$ ). Then, he was discharged but was still administered oral gliclazide $(0.8 \mathrm{mg} /$
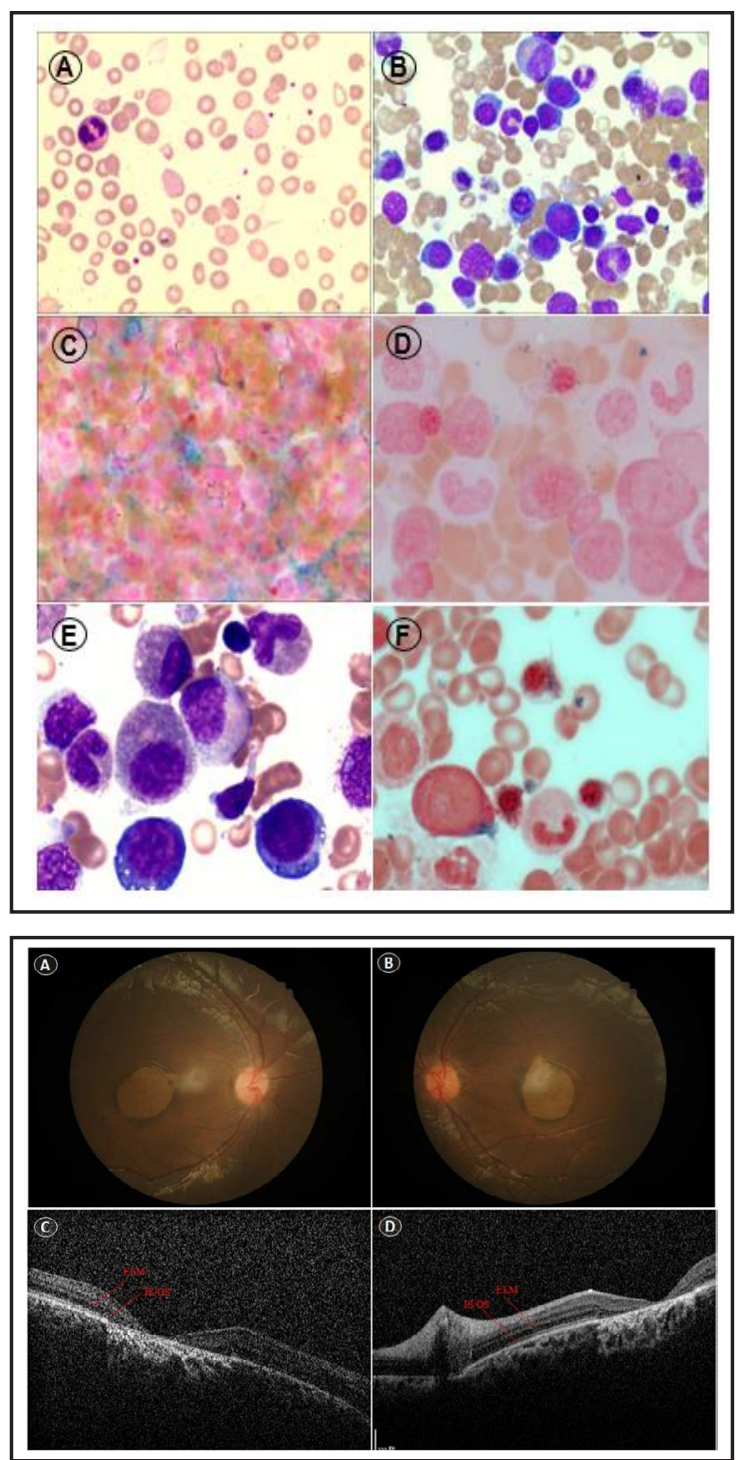

Fig. 2. Proband's eye examination. Color eye fundus images of the proband showed binocular macular defects (A, B) and optical coherence tomography showed a thinned retina at the macular center (at recession), with the disappearance of the ELM and IS/ OS layer (C, D). $\mathrm{kg} / \mathrm{d}$ ). His blood glucose level, however, varied within 12-19 mmol/L, indicating an unsatisfactory reduction. Subsequently, he was hospitalized in our hospital, and presented with skin and mucous pallor, minor bleeding and ecchymosis of the hand and foot, but no abnormalities in the heart, chest, neck, or head. His routine blood test indicated: (1) RBC: $1.86 \times 10^{12} / \mathrm{L}, \mathrm{Hb}: 54.0 \mathrm{~g} / \mathrm{L}, \mathrm{WBC}: 4.7 \times 10^{9} / \mathrm{L}$, platelets: $9 \times 10^{9} / \mathrm{L}, \mathrm{MCV}$ : $88 \mathrm{fl}$, and HCT: 0.21 ; (2) no functional abnormalities of the liver, kidney and electrolytes, FBG $13.9 \mathrm{mmol} / \mathrm{L}$ and LD $254 \mathrm{U} / \mathrm{L}$ by biochemical test; (3) the presence of an active hyperplasia and developmental disorder, indicated by megaloblastic degeneration and ring sideroblasts, decreased megakaryocyte production, and rare platelets in the bone marrow detected by bone marrow cytology (Fig. 1E); (4) extracellular iron (many iron particles and iron beads), intracellular iron 61\%, and ring sideroblasts $25 \%$ shown by iron staining (Fig. 1F). Considering these parameters, the proband's brother was suspected, 
but not diagnosed, with myelodysplastic syndrome combined with diabetes mellitus. After communication, his parents expressed an understanding of the situation, and the proband's brother was treated with a WBC/RBC-deleted blood transfusion and platelet treatment before discharge. Unfortunately, he died at 8 months of age.

As shown in Table 1, clinical manifestations and laboratory test results were compared between the proband and hisbrother. The proband was administered oral thiamine $(30 \mathrm{mg} / \mathrm{d})$ for $1-3$ months and exhibited normal $\mathrm{Hb}, \mathrm{FBG}$, insulin, and C-peptide levels, significantly decreased glycosylated hemoglobin (Table 2), as well as irreversible irreversible.

\section{Mutational analysis}

PCR sequences revealed a c.1409insT homozygous insertion mutation in exon 6 of SLC19A2 in the proband, and his parents and sister were confirmed as heterozygous carriers of c.1409insT mutations, as shown in Fig. 3. By retrieval, this mutation was confirmed absent from the NCBI SNP database (http://www. ncbi.nlm.nih.gov/SNP) (Fig. 4).

\section{Bioinformatics analysis results}

The transmembrane region of THTR1 was predicted by WEBSEQUENCE TMHMM2.0, and the results showed that amino acids 471-497 were localized intracellularly and in the cell membrane. (Fig. 5A). Partial amino acid sequences of THTR-1 in 10 species (human, pan troglodytes, nomascus leucogenys, macaca mulatta, bos taurus, sus scrofa, felis catus, canis lupus familiaris, mus musculus, rattus norvegicus) were compared, and contained part of the highly conserved amino acid in this mutant region (red). (Fig. 5B). The three-dimensional structure of THTR-1 was modeled using the I-TASSER server. The wild type sequence is shown in red and the c.1409insT mutant is shown in green. The results showed that the mutations caused a change in protein conformation (Fig. 5C).

\section{HPLC detection results}

As shown in Fig. 6, the retention times for thiamine, TMP the retention time of TPP in the whole blood was 6.1 minutes for the proband and healthy controls. Here, we used the peak area of TPP instead of the TPP relative concentration in the whole blood and ensured that all the numerical values were in the linear range of the test. There was a significant difference between the mean \pm SD peak area of TPP 
Fig. 4. Gene sequencing results in exon 6 of the SLC19A2 gene in a family with thiamine-responsive megaloblastic anemia. A c.1409insT homozygous insertion mutation in exon 6 of SLC19A2 was found in the proband, and his parents and sister were diagnosed as heterozygous carriers of c.1409insT mutations in the SLC19A2 gene. Red arrows indicate mutation sites.

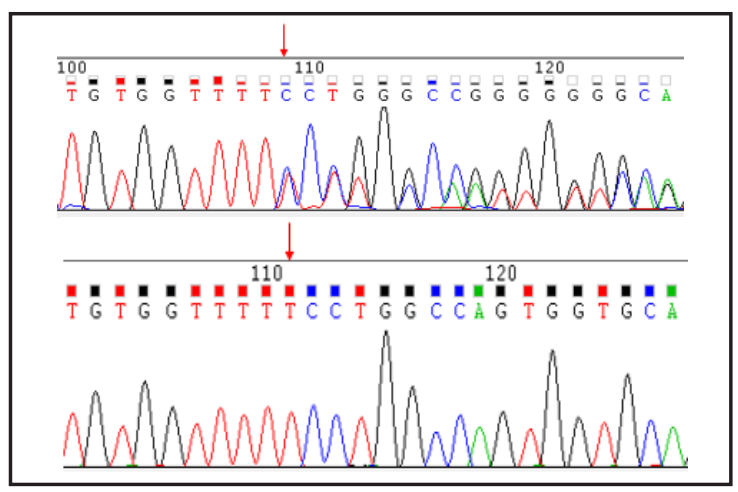

Fig. 5. Computer analysis and prediction of the protein conformation of mutant THTR-1. A. The transmembrane region of THTR- 1 was predicted by TMHMM Server v. 2.0, and the results showed that amino acids 471-497 were localized intracellularly and in the cell membrane. B. Partial amino acid sequences of THTR- 1 in 10 species were compared, and contained part of the highly conserved amino acid in this mutant region (red). C. The threedimensional structure of THTR-1 was modeled using the I-TASSER server. Wild type sequences are shown in red and c.1409insT mutants are shown in green. The results showed that the mutations caused a change in protein conformation.

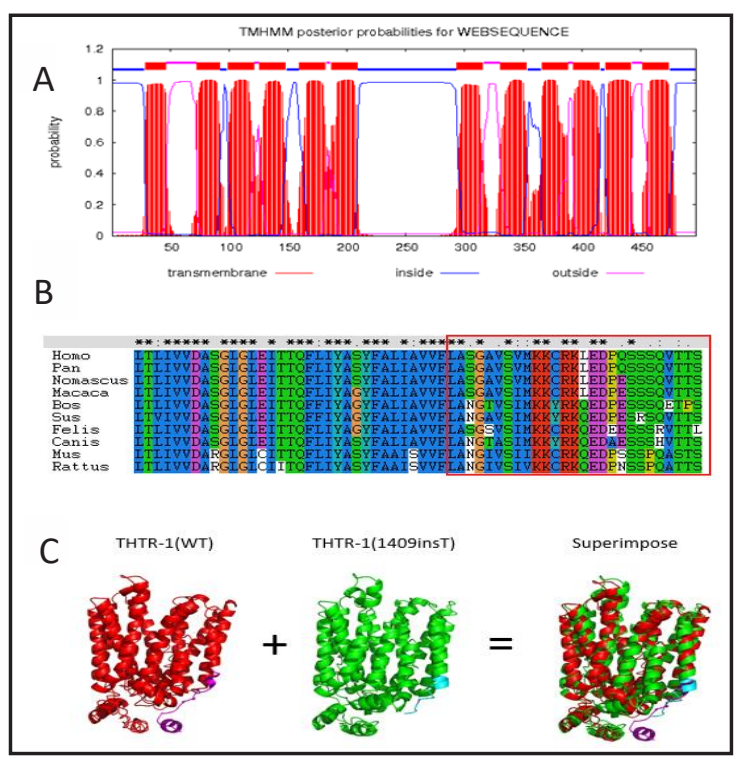

Fig. 6. High performance liquid chromatography results. The retention times of thiamine, thiamine monophosphate (TMP) and thiamine pyrophosphate (TPP) were 12.5, 7.8 and 6.6 minutes for the calibrator (A), a healthy control sample (B), the sample before supplementation with thiamine and after supplementation with thiamine for 3 months of proband (C, D).

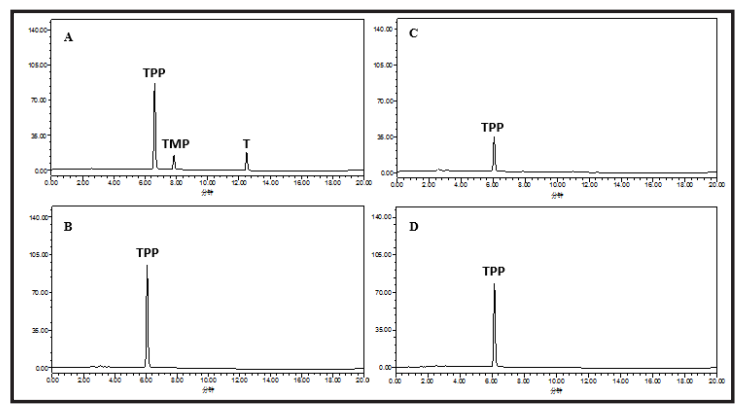

$\left(6545.00 \pm 706.62 \mathrm{mV}^{*} \mathrm{~s}\right)$ in the healthy control group and the proband $(2453.50 \pm 71.42$ $\mathrm{mV}^{*} \mathrm{~s}$ ) before the supplementation of thiamine ( $\left.\mathrm{P}=0.001\right)$. After supplementation with thiamine for 3 months, the peak area of TPP in the proband was increased to $6335 \pm 212.13$ $\mathrm{mV}^{*} \mathrm{~s}$. The proband's whole blood after thiamine supplement was significantly different $(\mathrm{P}=0.016)$ from that at baseline and was not significantly different from that in the healthy controls $(\mathrm{P}=0.711)$. The concentrations of thiamine and TMP in the whole blood were too low to be detected.

\section{Discussion}

In this study, a novel mutation in the SLC19A2 gene (c.1409insT) was found in a Chinese Zhuang ethnic family with TRMA syndrome with a homozygous mutation in the proband and 
heterozygous mutations in his parents and sister (Fig. 4). This mutation causes a reading frame shift in THTR-1 resulting in a shift in the position of a series of amino acids beginning at position 471. The transmembrane region of THTR-1 showed that amino acids 471-497 were localized intracellularly and in the cell membrane (Fig. 5A), which contained part of the highly conserved amino acid in this mutant region (Fig. 5B). Molecular modeling revealed this mutation resulted in a three-dimensional conformational change of the protein (Fig. 5C). We presume that this conformational change might disturb the implementation of the overall function of the THTR-1 protein.

THTR- 1 and THTR-2 are the major thiamine transport proteins expressed in most tissues. When THTR-1 is deficient, THTR-2 acts as a compensatory substitute to transport thiamine [7-10]. However, three THTR-1-dependent cells, including bone marrow cells, islet cells, and cochlear cells were reported to have no compensatory mechanisms and these eventually became dysfunctional [11]. This is the cytological basis of the three main clinical signs of TRMA, including anemia, diabetes, and progressive hearing loss. In this study, the proband appeared to have symptoms of anemia and diabetes, and the ABR threshold responding to the air conduction of the bilateral ears was $\leq 30 \mathrm{dBnHL}$, indicating normal hearing. The insertion mutation of THTR-1 does not cause hearing loss. May be the manifestation can occur at a later age. Even, if the proband represents a unique case of not having sensorineural hearing loss, which could be specific to the genotype.

In humans, thiamine exists in many forms, namely free thiamine (FT), TMP, TPP, thiamine triphosphate (TTP). About $90 \%$ of thiamine is in the TPP form, which is mainly distributed in erythrocytes. FT and TMP are mainly present in the plasma [12] and TTP in the central nervous system is difficult to detect in the peripheral blood because it is present at low levels. HPLC analysis of whole blood samples from healthy controls and the proband showed that the TPP retention time in the whole blood (6.1 minutes) was shorter than the standard value (6.6 minutes). This was presumably related to the complex components in the whole blood, or TPP in the cytoplasm bound to transketolase, resulting in a larger molecular weight of the conjugate and thereby causing a shorter TPP retention time by reverse HPLC. Thiamine is a water-soluble vitamin that remains in the body for only 3-6 hours, after which any excess is completely excreted, which might explain why we did not detect thiamine and TMP in the blood samples. This is consistent with the report of Talwar et al [13].. Under a normal diet, the proband showed a low concentration of TPP in whole blood when compared with healthy controls. After supplementation with thiamine for 3 months, the the relative concentration of TPP in the whole blood of the proband was increased, and no significant difference was found between the proband and healthy controls. In combination with clinical manifestations and gene mutation results, we speculated that erythrocytic TPP deficiency in the proband might be because of the SLC19A2 gene mutation causing THTR-1 dysfunction. TPP is a coenzyme for key enzymes in carbohydrate metabolism in vivo (transketolase, $\alpha$-ketoglutarate dehydrogenase, pyruvate dehydrogenase), and is involved in energy metabolism; therefore, TPP deficiency will directly lead to energy metabolism disorders.

Nutrient substances are considered essential for the development of RBCs. For example, a deficiency of folic acid and vitamin B12 results in erythrocytic nuclear alterations that trigger megaloblastic degeneration. Iron deficiency impinges on erythrocytic cytoplasm, accompanied by the emergence of erythroblasts with the retention of erythrocytic nuclei. Studies demonstrated the existence of megakaryocytes and ring sideroblasts in the bone marrow of TRMA patients [14], indicating that thiamine deficiency can simultaneously lead to abnormal development in the cytoplasm and nuclei. Thiamine is an important vitamin for humans, and its activated form is thiamine pyrophosphate, which is a coenzyme of transketolase in the phosphate pentose pathway. Thiamine pyrophosphate is involved in the hematopoietic synthesis of ribose 5-phosphate and its deficiency causes megaloblastic degeneration [15]. In addition, thiamine pyrophosphate is also a coenzyme of $\alpha$-ketoglutarate dehydrogenase involved in the synthesis of aminolevulinic acid, and its deficiency can reduce hemoglobin synthesis and cause iron utilization disorders, resulting in the emergence of ring sideroblasts [16]. This might explain the presence of megaloblastic erythrocytes (Fig.

\section{KARGER}


1B, Fig. 1E) and ring sideroblasts (Fig. 1D, Fig. 1F)in the bone marrow of the proband and his late brother investigated in the current study.

Previous studies reported that thiamine deficiency altered the secretion of pancreatic cells [17]. Rathanaswami et al [18]. reported isolated pancreatic islet cells from thiaminedeficient rats and reduced insulin, glucose and $\mathrm{CO}_{2}$ (produced by pyruvate oxidization) levels. Accordingly, they concluded that glucose oxidation was likely to reduce altered islet cell secretion. In the current study, the proband's brother was diagnosed with diabetes and treated with insulin in another hospital. During hospitalization, his blood glucose level varied between 5.8-12.0 $\mathrm{mmol} / \mathrm{L}$, but the level increased to $12-19 \mathrm{mmol} / \mathrm{L}$ after discharge, although he was given oral gliclazide. The proband's brother was not treated with thiamine, which is fundamental for TRMA patients. Diabetes in TRMA patients responds to the use of thiamine, because this activates the compensator transport of THTR-2 in beta cells. Gliclazide as secretagogues insulin may not act alone in TRMA patients.

Because of our lack of understanding regarding TRMA, we failed to define the pathogenesis of anemia in the proband's brother, and we presumed that he only suffered from TRMA. Multiple blood transfusions increased extracellular irons, accompanied by iron utilization disorders that resulted in a $25 \%$ increase in the number of ring sideroblasts (Fig. 1F). Therefore, the proband's brother was easily misdiagnosed as myelodysplastic syndrome. Considering the clinical manifestations of anemia, especially in children with suspected TRMA, it is important to determine first the etiology of anemia, upon which appropriate treatments can be developed. Clinicians can distinguish TRMA when confronted with full TRMA syndromes (macrocytic anemia, diabetes, progressive sensorineural hearing loss). However, incomplete symptoms may lead to a delayed diagnosis. The proband as an atypical clinical case of TRMA presented with macrocytic anemia, diabetes, but not deafness. Results from gene sequencing in the proband's family confirmed pathogenic genes and their mutation sites, providing evidence for TRMA diagnosis and treatment.

Supplementation with thiamine can improve the symptoms of anemia and diabetes in children with TRMA. Setoodeh et al [19]. reported four cases of children in Persia responsive to thiamine treatment. After early treatment, their $\mathrm{Hb}$ and HCT levels returned to normal, and their blood glucose levels were controlled. Ricketts et al [20]. found that thiamine supplementation became ineffective once puberty occurred. After adulthood, TRMA patients had to rely on blood transfusion and insulin therapy. Valerio et al [21]. performed a follow-up study of two children with TRMA. One child was subjected to oral glucose tolerance tests at 9 and 17.5 years of age, and the other at 13 and 17 years of age. By comparing the children's serum insulin levels at two growth stages, Valerio et al. observed reduced insulin secretion in these two children after puberty, suggesting that thiamine deficiency might cause islet cell apoptosis and reduce insulin secretion, indicating it was insufficient to meet the physiological needs of adolescence. Based on previous literature, we speculate that puberty is a critical period for the metabolic control of TRMA patients. In this study, the proband was diagnosed with TRMA, and then given oral thiamine $(30 \mathrm{mg} / \mathrm{d})$ for $1-3$ months. His $\mathrm{Hb}, \mathrm{FGB}$, insulin, and C-peptide levels recovered to normal, and his glycosylated hemoglobin level was significantly decreased (Table 2). However, the proband still suffered from macular defect and retinal atrophy (Fig. 2). Ach et al [22]. reported a case of TRMA with optic nerve atrophy that still had decreased vision and visual field and presented with "cattle eye" on color fundus imaging, following oral thiamine treatment for 5 years. OCT test results showed outer membrane damage, retinal epithelial cell irregularities and choroidal lesions in this case. These findings indicate that thiamine therapy may be unable to prevent and reverse visual impairment in TRMA patients. Currently, the proband is being followed-up for a long duration for the continuous evaluation of the efficacy of thiamine supplementation, which we will use to update his therapeutic regimen.

\section{Disclosure Statement}

The authors declare that no conflict of interests exists. 


\section{Cellular Physiology Cell Physiol Biochem 2018;47:1989-1997 \begin{tabular}{l|l} 
and Biochemistry & DOI: 10.1159/000491467 \\
Published online: July 03, 2018 & $\begin{array}{l}\text { (c) } 2018 \text { The Author(s). Published by S. Karger AG, Basel } \\
\text { www.karger.com/cpb }\end{array}$
\end{tabular}}

Xian et al.: Clinical Manifestations of a Novel Mutation of SLC19A2

\section{References}

1 Bergmann AK, Sahai I, Falcone JF, Fleming J, Bagg A, Borgna-Pignati C, Casey R, Fabris L, Hexner E, Mathews L, Ribeiro ML, Wierenga KJ, Neufeld EJ: Thiamine-responsive megaloblastic anemia: Identification of novel compound heterozygotes and mutation update. J Pediatr 2009;155:888-892 e881.

2 Aycan Z, Bas VN, Cetinkaya S, Agladioglu SY, Kendirci HN, Senocak F: Thiamine-responsive megaloblastic anemia syndrome with atrial standstill: A case report. J Pediatr Hematol Oncol 2011;33:144-147.

-3 Srikrupa NN, Meenakshi S, Arokiasamy T, Murali K, Soumittra N: Leber's congenital amaurosis as the retinal degenerative phenotype in thiamine responsive megaloblastic anemia: A case report. Ophthalmic Genet 2014;35:119-124.

4 Labay V, Raz T, Baron D, Mandel H, Williams H, Barrett T, Szargel R, McDonald L, Shalata A, Nosaka K, Gregory S, Cohen N: Mutations in slc19a2 cause thiamine-responsive megaloblastic anaemia associated with diabetes mellitus and deafness. Nat Genet 1999;22:300-304.

-5 Zhang Y: I-tasser server for protein 3d structure prediction. BMC Bioinformatics 2008;9:40.

6 Lu J, Frank EL: Rapid hplc measurement of thiamine and its phosphate esters in whole blood. Clin Chem 2008;54:901-906.

7 Fleming JC, Tartaglini E, Steinkamp MP, Schorderet DF, Cohen N, Neufeld EJ: The gene mutated in thiamineresponsive anaemia with diabetes and deafness (trma) encodes a functional thiamine transporter. Nat Genet 1999;22:305-308.

8 Rindi G, Ferrari G: Thiamine transport by human intestine in vitro. Experientia 1977;33:211-213.

-9 Laforenza U, Patrini C, Alvisi C, Faelli A, Licandro A, Rindi G: Thiamine uptake in human intestinal biopsy specimens, including observations from a patient with acute thiamine deficiency. Am J Clin Nutr 1997;66:320-326.

10 Hoyumpa AM, Jr., Strickland R, Sheehan JJ, Yarborough G, Nichols S: Dual system of intestinal thiamine transport in humans. J Lab Clin Med 1982;99:701-708.

$>11$ Reidling JC, Lambrecht N, Kassir M, Said HM: Impaired intestinal vitamin b1 (thiamin) uptake in thiamin transporter-2-deficient mice. Gastroenterology 2010;138:1802-1809.

$\checkmark 12$ Losa R, Sierra MI, Fernandez A, Blanco D, Buesa JM: Determination of thiamine and its phosphorylated forms in human plasma, erythrocytes and urine by hplc and fluorescence detection: A preliminary study on cancer patients. J Pharm Biomed Anal 2005;37:1025-1029.

13 Talwar D, Davidson H, Cooney J, St JRD: Vitamin b(1) status assessed by direct measurement of thiamin pyrophosphate in erythrocytes or whole blood by hplc: Comparison with erythrocyte transketolase activation assay. Clin Chem 2000;46:704-710.

14 Bazarbachi A, Muakkit S, Ayas M, Taher A, Salem Z, Solh H, Haidar JH: Thiamine-responsive myelodysplasia. Br J Haematol 1998;102:1098-1100.

15 Boros LG, Steinkamp MP, Fleming JC, Lee WN, Cascante M, Neufeld EJ: Defective rna ribose synthesis in fibroblasts from patients with thiamine-responsive megaloblastic anemia (trma). Blood 2003;102:35563561.

16 Abboud MR, Alexander D, Najjar SS: Diabetes mellitus, thiamine-dependent megaloblastic anemia, and sensorineural deafness associated with deficient alpha-ketoglutarate dehydrogenase activity. J Pediatr 1985;107:537-541.

17 Prasannan KG, Sundaresan R, Venkatesan D: Thiamine deficency and protein secretion by pancreatic slices in vitro. Experientia 1977;33:169-170.

18 Rathanaswami P, Pourany A, Sundaresan R: Effects of thiamine deficiency on the secretion of insulin and the metabolism of glucose in isolated rat pancreatic islets. Biochem Int 1991;25:577-583.

19 Setoodeh A, Haghighi A, Saleh-Gohari N, Ellard S, Haghighi A: Identification of a slc19a2 nonsense mutation in persian families with thiamine-responsive megaloblastic anemia. Gene 2013;519:295-297.

20 Ricketts CJ, Minton JA, Samuel J, Ariyawansa I, Wales JK, Lo IF, Barrett TG: Thiamine-responsive megaloblastic anaemia syndrome: Long-term follow-up and mutation analysis of seven families. Acta Paediatr 2006;95:99-104.

21 Valerio G, Franzese A, Poggi V, Tenore A: Long-term follow-up of diabetes in two patients with thiamineresponsive megaloblastic anemia syndrome. Diabetes Care 1998;21:38-41.

-22 Ach T, Kardorff R, Rohrschneider K: Fundus autofluorescence and optical coherence tomography findings in thiamine responsive megaloblastic anemia. Retin Cases Brief Rep 2015;9:114-116. 